\title{
The Role of Childhood Trauma and PTSD in Postpartum Sleep Disturbance
}

\author{
Leslie M. Swanson, Lindsay Hamilton, and Maria Muzik \\ Department of Psychiatry, University of Michigan, Ann Arbor, Michigan, USA
}

\begin{abstract}
Despite robust associations between postpartum sleep difficulties and maternal psychopathology, little attention has been paid to the role of childhood trauma and posttraumatic stress disorder (PTSD). In the present study, we examined sleep complaints in postpartum women with a history of childhood trauma compared to postpartum women who were not exposed to childhood trauma. Participants $(N=173)$ completed questionnaires by telephone at 4-months postpartum. After adjusting for nuisance variables, there were significantly higher rates of sleep disturbance (falling asleep and staying asleep) for women with a past history of neglect $(O R=4.84, p=.036$ and $5.78, p=.006$, respectively), physical abuse $(O R=9.20, p=.002$ and 3.84, $p=.044$, respectively), and physical abuse with sexual abuse $(O R=5.95$, $p=.011$ and 3.56, $p=.045$, respectively). Current PTSD was significantly associated with trouble staying asleep $(O R=4.21, p=.032)$ whereas recovery from PTSD was associated with trouble falling $(O R=4.19, p=.015)$ and staying asleep $(O R=3.69, p=.011)$. Our findings affirm the contribution of childhood trauma and PTSD to postpartum sleep.
\end{abstract}

Sleep disruption is normative in the postpartum period, even among healthy women (Gay, Lee, \& Lee, 2004; Signal et al., 2007). Nevertheless, difficulty sleeping at night when the infant is asleep can signal depression and anxiety in new mothers (Dørheim, Bondevik, Eberhard-Gran, \& Bjorvatn, 2009; Swanson, Pickett, Flynn, \& Armitage, 2011). Among factors that may influence sleep in the postpartum period, little attention has been paid to the role of childhood trauma, which is known to negatively affect sleep in nonperinatal adults (Bader et al., 2007; Chapman et al., 2013; Greenfield, Lee, Friedman, \& Springer, 2011). Further, childhood sexual abuse is a significant risk factor for the development of posttraumatic stress disorder (PTSD) in adult females (Tolin \& Foa, 2006). A recent prospective study identified a past history of sexual trauma as a major predictor of PTSD in postpartum women (Verreault et al., 2012). Females are at greater risk for developing PTSD after traumatic events relative to males (Tolin \& Foa, 2006), and estimates suggest that as many as $8 \%$ of women report significant symptoms of PTSD at 1-month postpartum (Verreault et al., 2012), a condition which is closely associated with poor sleep (Germain, 2013).

This research was supported by a grant from the National Institutes of Health (NIMH K23 MH080147) awarded to Maria Muzik.

Correspondence concerning this article should be addressed to Leslie $\mathrm{M}$. Swanson, Department of Psychiatry, University of Michigan, 4250 Plymouth Rd, Ann Arbor, MI 48109. E-mail: LMSwan@med.umich.edu

Copyright ( 2014 International Society for Traumatic Stress Studies. View this article online at wileyonlinelibrary.com

DOI: $10.1002 /$ jts. 21965
There is evidence thus far in the literature to suggest that experiencing trauma at other points in the lifespan is associated with sleep disruption. Sleep disruption is common after experiencing trauma (Mellman, Bustamante, Fins, Pigeon, \& Nolan, 2002), and may persist beyond the time frame of the initial event. Adult survivors of childhood trauma are more likely to experience a variety of sleep complaints relative to individuals who have not been exposed to childhood trauma, including a longer sleep-onset latency (Bader et al., 2007), poorer subjective sleep quality (Greenfield et al., 2011), insufficient sleep (Chapman et al., 2013), daytime sleepiness (Greenfield, et al., 2011), and insomnia (Bader et al., 2007).

Very few studies, however, have examined whether sleep is differentially affected by the type of childhood trauma experienced. One exception, a 2011 study by Greenfield et al., showed that childhood emotional and physical abuse, even in the absence of sexual abuse, was associated with disturbed sleep in adulthood. Further, much of the existing literature on sleep in adult survivors of childhood trauma does not take into account PTSD. A study of adolescent girls who experienced childhood sexual abuse suggested that sexual abuse was associated with sleep disturbance even with depression and PTSD in the model (Noll, Trickett, Susman, \& Putnam, 2006). Sleep disturbances are common in PTSD (Ohayon \& Shapiro, 2000). Indeed, sleep disruption following trauma is considered a hallmark of PTSD (Germain, 2013; Ross, Ball, Sullivan, \& Caroff, 1989). Importantly, insomnia is known to persist even in individuals who have recovered from PTSD (Zayfert \& DeViva, 2004).

In the present study, we sought to address gaps in the literature by examining sleep complaints in postpartum women with a history of childhood trauma compared to postpartum women 
without a history of childhood trauma. Our goals were to determine whether sleep was differentially affected by the type of childhood trauma women had experienced, and to understand the contribution of PTSD to sleep complaints. We hypothesized that having experienced any type of abuse or neglect in childhood would increase the risk of sleep disturbance regardless of the status of PTSD, and that a history of childhood abuse (sexual or physical) would be associated with the greatest risk for sleep complaints. We expected that mothers with PTSD, either current or past, would report more sleep disturbance than mothers who experienced childhood trauma, but did not develop PTSD.

\section{Method}

\section{Participants and Procedure}

The sample consisted of 173 women who were approximately 4-months postpartum, with a mean age of 28.26 years ( $S D=5.61$ years). Women were recruited on a continual basis as part of a longitudinal study where data were collected at several time points after birth. Women at this wave of the study were on average 4.72-months postpartum ( $S D=25.83$ days). Other demographic characteristics of the sample are summarized in Table 1 . The majority of participants $(80.1 \%)$ were

Table 1

Demographic Characteristics of the Sample

\begin{tabular}{|c|c|c|}
\hline Variable & $n$ & $\%$ \\
\hline \multicolumn{3}{|l|}{ Sex of infant ${ }^{\mathrm{a}}$} \\
\hline Male & 88 & 46.2 \\
\hline Female & 80 & 50.9 \\
\hline \multicolumn{3}{|l|}{ Race $^{\mathrm{b}}$} \\
\hline Caucasian & 104 & 60.1 \\
\hline African American & 42 & 24.3 \\
\hline Asian & 8 & 4.6 \\
\hline Hispanic & 4 & 2.3 \\
\hline Other & 7 & 4.0 \\
\hline \multicolumn{3}{|l|}{ Marital status ${ }^{\mathrm{c}}$} \\
\hline Married/partnered & 127 & 73.4 \\
\hline Never married & 28 & 16.2 \\
\hline Divorced/widowed & 3 & 1.7 \\
\hline \multicolumn{3}{|l|}{ Education $^{\mathrm{b}}$} \\
\hline Less than high school & 7 & 4.0 \\
\hline High school/GED & 22 & 12.7 \\
\hline Some college & 42 & 24.3 \\
\hline Bachelor's degree or higher & 94 & 54.3 \\
\hline \multicolumn{3}{|l|}{ Income $^{\mathrm{d}}(\$)$} \\
\hline$<15,000$ & 39 & 22.5 \\
\hline $15000-25,000$ & 19 & 11.0 \\
\hline $25000-50,000$ & 30 & 17.3 \\
\hline$>50,000$ & 82 & 47.4 \\
\hline
\end{tabular}

Note. $N=173$. GED $=$ General Educational Development Test.

${ }^{\mathrm{a}} n=168 .{ }^{\mathrm{b}} n=165 .{ }^{\mathrm{c}} n=158 .{ }^{\mathrm{d}} n=170$. primiparous. Sleep problems were relatively common across the sample; 44 (25.4\%) endorsed difficulty staying asleep and $39(22.5 \%)$ reported difficulty falling asleep. With respect to childhood trauma, 64 participants $(36.9 \%)$ reported that they did not experience any childhood neglect or abuse, and 109 $(63.0 \%)$ reported a history of childhood maltreatment. The most commonly reported types of childhood trauma included sexual abuse alone (35 women, $20.2 \%$ of the total sample), and both physical and sexual abuse (28 women, $16.2 \%$ of the total sample). Of the 109 participants who reported experiencing childhood neglect or abuse, 32 (29.4\%) never experienced PTSD; $53(48.6 \%)$ had recovered from a past history of PTSD; and $24(22.0 \%)$ had persistent PTSD. There were no cases of newonset PTSD following delivery: All women who met diagnostic criteria for PTSD at 4-months postpartum also met diagnostic criteria prior to pregnancy. Thirty participants $(17.3 \%$ of the sample) met criteria for current depression.

Data for this study were collected as part of the Maternal Anxiety during the Childbearing Years study (MACY study; NIMH K23 MH080147), a longitudinal investigation of the impact of maternal childhood adversity on postpartum functioning. Further detail regarding the parent study is published elsewhere (Muzik et al., 2013). For the present study, we utilized data collected at 4-months postpartum, the first wave of the study. Participants were recruited from pregnancy clinics in a midwestern metropolitan area and through flyers posted in the community. Although participants with difficult childhood experiences were oversampled, only $63 \%$ scored above cutoff scores for abuse or neglect using the Childhood Trauma Questionnaire (Bernstein \& Fink, 1998). Participants were consented verbally over the phone at 4 months. All participants were 18 years of age or older, nonpsychiatrically referred, and English speaking. Exclusion criteria included the use of illegal or nonprescription drugs during pregnancy, maternal history of bipolar and psychotic illness, child prematurity $(<36$-weeks term), or child developmental disability or severe illness (e.g., epilepsy). Women who met criteria for lifetime PTSD during the assessment phone call, but did not meet abuse or neglect criteria for childhood maltreatment, were excluded from the study. The study was approved by the University of Michigan Medical School Institutional Review Board. Potential participants were screened through an initial telephone interview to determine study eligibility. Data collected by telephone at 4-months postpartum consisted of a demographics questionnaire, childhood trauma assessment, and psychiatric assessment, including diagnosis of current and lifetime (prepregnancy) PTSD.

\section{Measures}

Demographic information included age, race, income, education, marital status, parity, and body mass index (BMI). The Life Stressor Checklist (Wolfe \& Kimerling, 1997) was used to assess history of traumatic events. The diagnosis of PTSD was made using the National Women's Study (NWS) PTSD module (Resnick, Kilpatrick, Dansky, Saunders, \& Best, 1993), 
utilizing criteria outlined in the Diagnostic and Statistical Manual of Mental Disorders (4th ed.; DSM-IV; American Psychiatric Association, 1994). The module is a structured telephone diagnostic interview designed to be administered by lay interviewers, with a sensitivity of .99 and specificity of .79 to detect PTSD diagnosis (Resnick et al., 1993). Cronbach's $\alpha$ for this measure was .88 in our sample. For this study, women were asked about prepregnancy symptoms to assess for lifetime PTSD. Women were asked to report on lifetime PTSD symptoms prior to pregnancy or during pregnancy until childbirth, and about current PTSD since the birth of the child (i.e., past 4 months). Participants were characterized as follows: no PTSD (neither lifetime nor current diagnosis), recovered PTSD (lifetime history of PTSD, but not meeting current diagnosis in past 4 months), and persistent PTSD (lifetime history and current diagnosis of PTSD). Participants in the no PTSD and recovered PTSD categories could have subthreshold symptoms of PTSD, but did not have enough symptoms to meet diagnostic criteria for a current diagnosis of PTSD.

Depression was measured using the Postpartum Depression Screening Scale (PDSS; Beck \& Gable, 2002), which assesses risk for postpartum depression. The measure assesses seven domains related to depression over the past 2 weeks. Total scores of 80 or greater were considered positive for a diagnosis of major postpartum depression per DSM-IV criteria (American Psychiatric Association, 1994). The PDSS has a sensitivity of .94 and specificity of .98 for major depression at this cut point. Cronbach's $\alpha$ for the PDSS was .96 in the present study.

Childhood trauma history was assessed using the Childhood Trauma Questionnaire (CTQ; Bernstein \& Fink, 1998). The CTQ is a validated, retrospective measure of traumatic childhood experiences. For the present study, participants were categorized as follows: reported no childhood abuse or neglect; reported childhood neglect (emotional or physical), but did not report any abuse; reported childhood physical abuse (with or without neglect); reported childhood sexual abuse (with or without neglect); or reported both childhood physical and sexual abuse (with or without neglect).

Maternal sleep complaints were also determined from the PDSS. Sleep items, assessed over the past 2 weeks, included trouble staying asleep ("You woke up on your own in the middle of the night and had trouble getting back to sleep"), and trouble falling asleep ("You tossed and turned for a long time at night trying to fall asleep"). Items are scored on a Likert-type scale, from $1=$ strongly disagree to $5=$ strongly agree. As we were interested in whether childhood trauma and PTSD were associated with any level of sleep disturbance, participants who indicated they agreed or strongly agreed were categorized as experiencing difficulty in that sleep domain. Thus, sleep outcomes were binary (i.e., participants were categorized as either positive or negative for each sleep complaint).

Sleep in the infant was measured using a composite Cry, Feed and Sleep Questionnaire (CFS; Seifer, 1996), an 86 item parent-report measure adapted from the Children's Sleep Habits Questionnaire (CSHQ; Owens, Spirito, \& McGuinn, 2000). The
CFS includes items on infant sleep and waking, feeding habits, and crying patterns over the past week. The CSHQ is a validated measure. Cronbach's $\alpha$ for the CFS in the present study was .67. The following domains, identified by Seifer (1996), were used to characterize infant sleep: bedtime behavior, sleep behaviors, nighttime awakenings, morning awakening, and daytime sleepiness.

\section{Data Analysis}

All data analyses were performed using SPSS version 17. All analyses used two-sided tests with a significance level of .05. With respect to missing data, two participants had missing data for one item on the PDSS. Neither of these items were sleep items and both participants scored above the depression cutpoint even with the missing item, thus their categorization for the purposes of the present study was not affected by this missing data. Nine participants were missing data for one or more items on the NWS PTSD module; however, all of these participants had sufficient complete data to ascertain PTSD diagnosis regardless of the missing items.

Correlations between demographic variables and outcome variables were calculated. Odds ratios were calculated using logistic regression analyses, one to determine whether after including a diagnosis of PTSD and nuisance variables in the model, women with a history of childhood neglect or abuse were more likely to report a significant sleep complaint than women without a history of childhood trauma, and a second set of analyses to test the contribution of PTSD to sleep difficulties.

To determine the nuisance variables for the logistic regression models, stepwise regressions were conducted for each dependent variable using the following variables: number of children, breastfeeding status, birth complications, preterm delivery, infant medical conditions or disability at birth, maternal BMI, maternal depression diagnosis, child gender, race, age, income, education, marital status, and infant sleep domains measured on the CFS. Only those variables retained in the stepwise regression model were used in the logistic regressions.

\section{Results}

Stepwise regression analyses, conducted to determine nuisance variables, indicated that depression diagnosis was associated with difficulty falling asleep and difficulty staying asleep. Additionally, difficulty staying asleep was associated with infant nighttime awakenings. Logistic regression analyses were adjusted for these variables.

Of the demographic variables, only marital status was significantly correlated with sleep (difficulty staying asleep; $\varphi=.27$, $p=.025)$. Significant correlations were observed between difficulty falling asleep and depression diagnosis $(\varphi=.48, p<.001)$ and PTSD diagnosis $(\varphi=.30, p<.001)$. Difficulty staying asleep was correlated with depression diagnosis $(\varphi=.43$, $p \leq .001)$ and PTSD diagnosis $(\varphi=.30, p<.001)$.

With the additional variables in the model, participants who reported childhood neglect, physical abuse, and physical abuse 
Table 2

Adjusted Odds Ratios for Experiencing a Sleep Complaint by Childhood Trauma History

\begin{tabular}{|c|c|c|c|c|c|c|c|c|c|}
\hline \multirow[b]{3}{*}{ Type of childhood trauma } & \multirow[b]{3}{*}{$N^{\mathrm{a}}$} & \multicolumn{8}{|c|}{ Sleep complaint } \\
\hline & & \multicolumn{4}{|c|}{ Falling asleep } & \multicolumn{4}{|c|}{ Staying asleep } \\
\hline & & $n$ & $\%$ & $A O R^{\mathrm{b}}$ & $95 \% \mathrm{CI}$ & $n$ & $\%$ & $A O R^{\mathrm{c}}$ & $95 \% \mathrm{CI}$ \\
\hline No history of neglect or abuse $\mathrm{d}^{\mathrm{d}}$ & 64 & 5 & 7.8 & 1.00 & & 7 & 10.9 & 1.00 & \\
\hline Neglect (emotional or physical) only & 21 & 6 & 28.6 & $4.84^{*}$ & {$[1.11,21.19]$} & 9 & 42.9 & $5.78^{* *}$ & {$[1.65,20.23]$} \\
\hline Physical abuse ( \pm neglect) & 25 & 11 & 44.0 & $9.20^{* *}$ & {$[2.20,38.45]$} & 10 & 40.0 & $3.84^{*}$ & {$[1.04,14.24]$} \\
\hline Sexual abuse ( \pm neglect $)$ & 35 & 7 & 20.0 & 1.21 & {$[0.28,5.25]$} & 8 & 22.8 & 1.06 & {$[0.29,3.87]$} \\
\hline Physical and sexual abuse ( \pm neglect) & 28 & 10 & 35.7 & $5.95^{*}$ & {$[1.50,23.57]$} & 10 & 35.7 & $3.56^{*}$ & {$[1.03,12.31]$} \\
\hline
\end{tabular}

Note. $A O R=$ adjusted odds ratio; $\mathrm{CI}=$ confidence interval; $\mathrm{PTSD}=$ posttraumatic stress disorder.

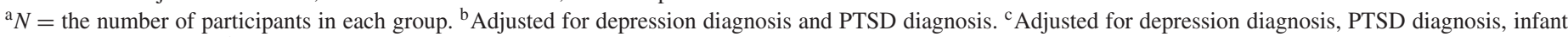
nighttime awakenings. ${ }^{\mathrm{d}}$ Reference group.

$* p<.05$. ** $p \leq .01$.

accompanied by sexual abuse were significantly more likely to endorse difficulty falling asleep and staying asleep (see Table 2) compared to participants who did not report a history of childhood abuse or neglect. Sexual abuse without physical abuse was not associated with increased odds for a sleep complaint.

As shown in Table 3, mothers who experienced childhood trauma but never developed PTSD had sleep profiles similar to women who were not exposed to childhood trauma. Mothers with a history of childhood trauma who had recovered from PTSD had greater odds of problems with falling and staying asleep. With all variables in the model, persistent PTSD was associated only with difficulty staying asleep.

\section{Discussion}

The aim of the present study was to examine relationships between sleep, childhood trauma exposure, and PTSD in a sample of newly postpartum women. We hypothesized that experiencing any childhood neglect or abuse would be associated with increased sleep disturbance, with women with a past history of childhood physical or sexual abuse at greatest risk. We also hypothesized that PTSD would be associated with the most sleep disturbance in the postpartum period.

Across our sample, one quarter of women reported sleep disturbance at 4-months postpartum. Notably, rates of sleep disturbance among our subsample of women without a past history of neglect or abuse were low, around $10 \%$. Significantly higher rates of sleep disturbance (both falling asleep and staying asleep) were observed for women with a past history of neglect, physical abuse, and physical abuse with sexual abuse, even after controlling for current PTSD and depression.

When we examined the contribution of PTSD to sleep complaints, we found that after adjusting for depression, difficulty staying asleep was significantly associated with persistent PTSD. Trouble maintaining sleep may be more salient to PTSD than sleep-onset difficulties, perhaps due to nightmares, a symptom unique to PTSD that can disrupt sleep in the middle of the night. Women who had recovered from a past history of PTSD were at nearly fourfold risk for experiencing difficulty falling and staying asleep relative to women who did not report

Table 3

Adjusted Odds Ratios for Experiencing a Sleep Complaint by PTSD Category

Sleep complaint

\begin{tabular}{|c|c|c|c|c|c|c|c|c|c|}
\hline \multirow[b]{3}{*}{ PTSD category } & \multirow[b]{3}{*}{$N^{\mathrm{a}}$} & \multirow{2}{*}{\multicolumn{4}{|c|}{ Falling asleep }} & & & & \\
\hline & & & & & & \multicolumn{4}{|c|}{ Staying asleep } \\
\hline & & $n$ & $\%$ & $A O R^{\mathrm{b}}$ & $95 \% \mathrm{CI}$ & $\bar{n}$ & $\%$ & $A O R^{\mathrm{c}}$ & $95 \% \mathrm{CI}$ \\
\hline No neglect/abuse history, no PTSD ${ }^{d}$ & 64 & 5 & 7.8 & 1.00 & & 7 & 10.9 & 1.00 & \\
\hline Neglect or abuse history, no PTSD & 32 & 6 & 18.8 & 3.02 & {$[0.79,11.54]$} & 5 & 15.6 & 1.62 & {$[0.45,5.83]$} \\
\hline Recovered PTSD & 53 & 15 & 28.3 & $4.19^{*}$ & {$[1.32,13.37]$} & 18 & 34.0 & $3.69^{*}$ & {$[1.35,10.10]$} \\
\hline Persistent PTSD & 24 & 13 & 54.1 & 4.04 & {$[0.97,16.82]$} & 14 & 58.3 & $4.21^{*}$ & {$[1.13,15.67]$} \\
\hline
\end{tabular}

Note. $A O R=$ adjusted odds ratios; $\mathrm{CI}=$ confidence interval; $\mathrm{PTSD}=$ posttraumatic stress disorder.

${ }^{\mathrm{a}} N=$ the number of participants in each group. ${ }^{\mathrm{b}}$ Adjusted for depression diagnosis. ${ }^{\mathrm{c}}$ Adjusted for depression diagnosis and infant nighttime awakenings. ${ }^{\mathrm{d}}$ Reference group.

${ }^{*} p<.05$. 
a history of childhood trauma. This extends work from previous studies that have shown that sleep problems persist after PSTD treatment and recovery (Zayfert \& DeViva, 2004). Importantly, women who reported a history of childhood trauma but never developed PTSD were not more likely to experience difficulty with sleep onset or maintenance relative to women who did not have a history of childhood trauma. Thus, in our sample of new mothers, those women who were resilient to PTSD had sleep that was similar to women who were never exposed to childhood trauma. This finding lends support to the hypothesis that sleep disturbances are a marker for compromised resilience to psychopathology, as recently posited by Germain (2013).

Contrary to our hypothesis, women who endorsed a childhood history of sexual abuse in the absence of physical abuse were not more likely to report difficulty with sleep. The scant literature on distal consequences of sexual abuse on sleep indicates that childhood sexual abuse is associated with sleep disturbance in adolescent girls, even with PTSD and depression (Noll et al., 2006) in the analysis. Differences between the frequency, severity, and type of sexual abuse experienced may account for the discrepancy in our findings, as the majority of our participants reported one-time sexual abuse perpetrated by a stranger, in contrast to Noll et al.'s (2006) sample, who had experienced sexual abuse perpetrated by a family member. We also note that our findings are consistent with Greenfield et al.'s (2011) work, which showed that childhood emotional and physical abuse, even in the absence of sexual abuse, is associated with poor sleep in nonperinatal adults.

This study has several limitations. First, our assessment of sleep was based on two self-report items drawn from a larger depression scale. These items provided a broad assessment of whether participants believed they were experiencing difficulty falling and staying asleep, without precise information that may further inform our understanding of the severity of their sleep problems. Thus, the low specificity of our sleep assessment is a limitation. Second, our cross-sectional design does not permit understanding of causal relationships between sleep and mood. Third, rates of revictimization were too low in our sample to permit analysis of whether additional traumas experienced in adulthood affected sleep. Finally, the study relied on telephonebased interviews, which may not be as accurate as face-toface clinical interviews. Further, diagnoses may not overlap perfectly between these two methods.

Future directions for this line of research include longitudinal designs, particularly studies that follow women with a history of childhood trauma through the childbearing period to understand relationships between mental health and sleep patterns during pregnancy and through the postpartum. Use of more precise measurements of sleep, including objective methods, will be important to fully appreciate how sleep in new mothers is affected by childhood trauma. Our understanding of how cumulative trauma affects sleep in perinatal women can be furthered by studies specifically focused on women who have experienced trauma in both childhood and adulthood.
To our knowledge, this is the first study of relationships between sleep, childhood trauma, and PTSD in a population that is particularly vulnerable to both sleep disturbance and PTSD. Our findings highlight the lasting effects of childhood trauma, particularly neglect and physical abuse, on sleep in new mothers. They also suggest that new mothers who have PTSD are likely to experience struggles with their sleep, which may in turn affect their daytime functioning and parenting capabilities. As our findings suggest that sleep symptoms may not resolve even after PTSD is in remission, the development of treatment strategies to address sleep in this population is critical.

\section{References}

American Psychiatric Association. (1994). Diagnostic and statistical manual of mental disorders (4th ed.). Washington, DC: Author

Bader, K., Schafer, V., Schenkel, M., Nissen, L., \& Schwander, J. (2007). Adverse childhood experiences associated with sleep in primary insomnia. Journal of Sleep Research, 16, 285-296. doi:10.1111/j.13652869.2007.00608.x

Beck, C. T., \& Gable, R. K. (2002). Postpartum Depression Screening Scale. Los Angeles, CA: Western Psychological Services.

Bernstein, D., \& Fink, L. (1998). Childhood Trauma Questionnaire: A retrospective self-report. San Antonio, TX: Psychological Corporation.

Chapman, D. P., Liu, Y., Presley-Cantrell, L. R., Edwards, V. J., Wheaton, A. G., Perry, G. S., \& Croft, J. B. (2013). Adverse childhood experiences and frequent insufficient sleep in 5 U.S. States, 2009: A retrospective cohort study. BMC Public Health, 13, 3. doi:10.1186/1471-2458-13-3

Dørheim, S., Bondevik, G., Eberhard-Gran, M., \& Bjorvatn, B. (2009). Sleep and depression in postpartum women: A population-based study. Sleep, 32, $847-855$.

Gay, C. L., Lee, K. A., \& Lee, S. Y. (2004). Sleep patterns and fatigue in new mothers and fathers. Biological Research for Nursing, 5, 311-318. doi:10.1177/1099800403262142

Germain, A. (2013). Sleep disturbances as the hallmark of PTSD: Where are we now? The American Journal of Psychiatry, 170, 372-382. doi:10.1176/appi.ajp.2012.12040432

Greenfield, E. A., Lee, C., Friedman, E. L., \& Springer, K. W. (2011). Childhood abuse as a risk factor for sleep problems in adulthood: Evidence from a U.S. national study. Annals of Behavioral Medicine, 42, 245-256. doi:10.1007/s12160-011-9285-x

Mellman, T. A., Bustamante, V., Fins, A. I., Pigeon, W. R., \& Nolan, B. (2002). REM sleep and the early development of posttraumatic stress disorder. American Journal of Psychiatry, 159, 1696-1701. doi:10.1176/appi.ajp.159.10.1696

Muzik, M., Bocknek, E. L., Broderick, A., Richardson, P., Rosenblum, K. L., Thelen, K., \& Seng, J. S. (2013). Mother- infant bonding impairment across the first 6 months postpartum: The primacy of psychopathology in women with childhood abuse and neglect histories. Archives of Women's Mental Health, 16, 29-38. doi:10.1007/s00737-012-0312-0

Noll, J. G., Trickett, P. K., Susman, E. J., \& Putnam, F. W. (2006). Sleep disturbances and childhood sexual abuse. Journal of Pediatric Psychology, 31, 469-480. doi:10.1093/jpepsy/jsj040

Ohayon, M. M., \& Shapiro, C. M. (2000). Sleep disturbances and psychiatric disorders associated with posttraumatic stress disorder in the general population. Comprehensive Psychiatry, 41, 469-478. doi:10.1053/comp.2000.16568 
Owens, J. A., Spirito, A., \& McGuinn, M. (2000). The Children's Sleep Habits Questionnaire (CSHQ): Psychometric properties of a survey instrument for school-aged children. Sleep, 23, 1043-1052.

Resnick, H. S., Kilpatrick, D. G., Dansky, B. S., Saunders, B. E., \& Best, C. L. (1993). Prevalence of civilian trauma and posttraumatic stress disorder in a representative national sample of women. Journal of Consulting and Clinical Psychology, 61, 984-991. doi:10.1037/0022-006X.61.6.984

Ross, R. J., Ball, W. A., Sullivan, K. A., \& Caroff, S. N. (1989). Sleep disturbance as the hallmark of posttraumatic stress disorder. American Journal of Psychiatry, 146, 697-707.

Seifer, R. (1996). Parental psychopathology and sleep variation in children. Child and Adolescent Psychiatric Clinics of North America, 5, 715727.

Signal, T. L., Gander, P. H., Sangalli, M. R., Travier, N., Firestone, R. T., \& Tuohy, J. F. (2007). Sleep duration and quality in healthy nulliparous and multiparous women across pregnancy and post-partum. Australian and New Zealand Journal of Obstetrics and Gynaecology, 47, 16-22. doi:10.1111/j.1479-828X.2006.00672.x
Swanson, L. M., Pickett, S. M., Flynn, H., \& Armitage, R. (2011). Relationships among depression, anxiety, and insomnia symptoms in perinatal women seeking mental health treatment. Journal of Women's Health, 20, 553-558. doi:10.1089/jwh.2010.2371

Tolin, D. F., \& Foa, E. B. (2006). Sex differences in trauma and posttraumatic stress disorder: A quantative review of 25 years of research. Psychological Bulletin, 132, 959-992. doi:10.1037/0033-2909.132.6.959

Verreault, N., Da Costa, D., Marchand, A., Ireland, K., Banack, H., Dritsa, M., \& Khalife, S. (2012). PTSD following childbirth: A prospective study of incidence and risk factors in Canadian women. Journal of Psychosomatic Research, 73, 257-263. doi:10.1016/j.jpsychores.2012.07.010

Wolfe, J., \& Kimerling, R. (1997). Gender issues in the assessment of PTSD. In J. P. Wilson \& T. M. Keane (Eds.), Assessing psychological trauma and posttraumatic stress disorder: A handbook for practitioners (pp. 192-219). New York, NY: Guilford Press.

Zayfert, C., \& DeViva, J. C. (2004). Residual insomnia following cognitive behavioral therapy for PTSD. Journal of Traumatic Stress, 17, 69-73. doi:10.1023/B:JOTS.0000014679.31799.e7 